\title{
Evaluation of Sphingomonas paucimobilis as an emerging nosocomial pathogen in a teaching hospital in Uttarakhand
}

\author{
Ranjana Rohilla $^{1}$, Dimple Raina ${ }^{1 *}$, Malvika Singh ${ }^{1}$, Ajay Kumar Pandita ${ }^{2}$, Shiwang Patwal ${ }^{1}$ \\ ${ }^{I}$ Department of Microbiology, Shri Guru Ram Rai Institute of Medical and Health Sciences, Dehradun, India \\ ${ }^{2}$ Department of Community Medicine, Shri Guru Ram Rai Institute of Medical and Health Sciences, Dehradun, \\ India
}

Received: June 2021, Accepted: August 2021

\begin{abstract}
Background and Objectives: Sphingomonas paucimobilis is an opportunistic pathogen and was rarely encountered in clinical specimens previously. This study aimed to investigate the clinical features, associated co-morbidities, and antimicrobial susceptibility patterns of $S$. paucimobilis infection in a tertiary hospital in Uttarakhand.

Materials and Methods: S. paucimobilis isolates cultured from various sections of hospital and OPDs were identified and analyzed for their antibiograms in the microbiology laboratory for a duration of one year from January 2020 to December 2020. Results: S. paucimobilis was isolated from 49 samples $(0.01 \%)$ out of 3792 samples processed in VITEK 2 Compact automated ID/AST instrument. The maximum number of isolates were obtained from urine samples (31\%), followed by blood (24\%). Septicemia (41\%), meningitis (17\%), lower respiratory tract infections and ventilator associated pneumonia (14\%) constituted a major portion of infections caused by this organism. Diabetes mellitus (22\%) and steroid usage (16\%) were major associated co-morbid conditions. Third and Fourth generation cephalosporins like ceftriaxone (81\%) and cefepime (86\%) were found to be the most susceptible drugs whereas $61 \%$ of isolates were resistant to colistin.

Conclusion: This organism is an up-and-coming pathogen and should not be simply labeled as a contaminant. Although the organism is not grossly virulent and still might not be associated with serious life-threatening infections; however their evolving resistance patterns and increased spectrum of infections should be seriously taken into account.
\end{abstract}

Keywords: Sphingomonas; Nosocomial; paucimobilis; Septicemia; Antimicrobial; Steroid

\section{INTRODUCTION}

Sphingomonas paucimobilis, an emergent pathogenic bacterium is aerobic, motile, non-fermenting Gram-negative bacilli. It is being implicated as growing cause of health care associated infections in hospitals in recent years (1). It is catalase and oxidase-positive and also exhibits the production of a yellow pigment (2). The organism is present everywhere and has been isolated from natural environmental conditions like seawater, sea ice, river water, wastewater, mineral water, and soil. It has a peerless sphingoglycolipid in the cell wall and lacks the lipopolysaccharide constituent in conjunction with its endotoxin activity. This could be the explanation for the low virulence of this organism (3). In fact, this organism can propagate in hemodialysis devices, nebulizers, ventilators, sterile drug solutions and distilled water (4). Both nosocomial and community-acquired infections can be caused by this organism; which were sparse previously but now their occurrence, particularly in hospital environment has augmented

*Corresponding author: Dimple Raina, MD, Department of Microbiology, Shri Guru Ram Rai Institute of Medical and Health Sciences, Dehradun, India. Tel: +91-9720170535Ｅmail: dr.dimpleraina@gmail.com 
(2). Blood, sputum, urine, wound, bile, cerebrospinal fluid, vagina, and cervix infections have been reported by this organism (5). Sphingomonas has plethora of resistant phenotypes, contributing to resistance to many antimicrobial agents tested. Individualized and appropriate antimicrobial therapy may need to be given to every patient (6). S. paucimobilis usually shows susceptibility to carbapenems, aminoglycosides, trimethoprim-sulfamethoxazole, and piperacillin/tazobactam whereas resistant patterns are majorly reported for penicillins and first-generation cephalosporins (7).

Till recent times $S$. paucimobilis was seldom encountered in the clinical milieu especially in immunocompromised patients and those with co-morbidities, however now there are many case reports to support the mounting body of evidence that this may be a budding infectious pathogen creditable of further probe and investigation (8). It is an emerging pathogen that should be dealt with cautiously $(8,9)$.

Thus, the current study aims to observe the clinical features of patients with $S$. paucimobilis infections and the antimicrobial susceptibility pattern of the isolated strains among the hospitalized patients in a tertiary health care institute for a duration of one year.

\section{MATERIALS AND METHODS}

A prospective study was conducted in the Department of Microbiology, Shri Guru Ram Rai Institute of Medical and Health Sciences; a tertiary care teaching hospital from January 2020 to December 2020. A study protocol was designed, written consent was taken from all the study participants, and approval was sought by the ethical and research committee of the institution.

Inclusion criteria was as follows: All clinical specimens from the patients attending outpatient departments (OPDs) and those admitted in the wards and ICUs of Shri Mahant Indiresh Hospital (SMIH), Patel Nagar Dehradun. Exclusion criteria was as follows: Unacceptable sample as per laboratory protocol and repeat samples from the same patient. Various clinical samples from patients like urine, pus, wound swabs, blood, other sterile fluids, respiratory specimens like Broncho-alveolar lavage (BAL), sputum, central line tips, bile, and cerebrospinal fluid (CSF) were analyzed in the Department of Microbiology.
After receiving specimens in the laboratory under all aseptic precautions, processing and reporting was done as per the standard methods. Microscopic examination was done by direct Gram staining followed by their culture onto Sheep Blood agar (5\%) and MacConkey agar \pm Chocolate agar except for blood specimens. Blood specimens were first inoculated in culture bottles of the automated blood culture system (BacT/Alert system, Organon Teknika Corporation, Durham, NC) followed by aerobic incubation for 5 days. If bottles flagged positive, subculture was taken on Blood agar and MacConkey agar. The inoculated plates were incubated aerobically at $37^{\circ} \mathrm{C}$ for 48 hours. For primary identification basic microbiological features and biochemical characteristics such as colony morphology, Gram staining, catalase, and oxidase tests were taken into account. Automated system (VITEK-2 Biomerieux) was used to detect bacteria from clinical specimens and the final identification and antibiotic susceptibility testing was reported based on it.

Data was entered and analyzed on Microsoft Excel and interpreted by descriptive methods in terms of frequency distribution in percentages, proportions, rates, ratios etc. Non-parametric tests i.e. chi-square tests were applied to ascertain the significance of the association.

\section{RESULTS}

In the study duration of one year, the total clinical samples received in the laboratory were 96,309. Significant growth was processed in 3792 samples using VITEK 2 Compact automated ID/AST instrument. S. paucimobilis was isolated in 1.3\% (49/3792) of samples. 23 out of 49 (46.9\%) patients had history of taking antibiotics before presenting to the hospital. 19 patient had devices on them (foley's catheter (8), endotracheal tube (6), central venous catheter in-situ (2), ventriculo-peritoneal shunt (1), intercostal drainage tube (1), and hemodialysis catheter (1)) but none had prosthetic implant.

Table 1 shows the distribution of $S$. paucimobilis isolates according to gender and age. Clinical manifestations of these patients $(n=49)$ were as illustrated in Fig. 1. Table 2 Shows distribution of samples from which $S$. paucimobilis was isolated $(\mathrm{n}=49)$. Table 3 shows the frequency distribution of $S$. paucimobilis isolated from various wards. The maximum 
number of isolates were obtained from and Intensive care units (ICUs) and High dependency units (HDUs) $(51 \%)$, followed by various wards $(37 \%)$ and outpatient departments (12\%). Diabetes $(22 \%)$ and steroid use $(16 \%)$ were the most frequent associated co-morbid conditions (Table 4). Table 5 depicts antibiotic

Table 1. Demographic parameters of the patients in whom Sphingomonas paucimobilis was isolated $(\mathrm{n}=49)$

\begin{tabular}{lcc}
\hline Demographics & Particulars & Number $(\%)$ \\
\hline Gender $(\mathrm{n}=49)$ & Male & $28(58 \%)$ \\
& Female & $21(42 \%)$ \\
Age in years $(\mathrm{n}=49)$ & $<20$ & $3(6 \%)$ \\
& $21-40$ & $14(29 \%)$ \\
$41-60$ & $26(53 \%)$ \\
& $>60$ & $6(13 \%)$
\end{tabular}

susceptibility patterns of isolates to various antibiotics agents (as per MIC breakpoints given in CLSI 2021 Guidelines) (10). Table 6 shows distribution of Multi-drug resistant and Extensively drug resistant isolated in various critical and non-critical area of the hospital. Of the 49 patients, 3 patients expired (6.1\%). All other cases $(93.8 \%$; 46/49) were successfully managed with antibiotics and were followed up as outpatients.

\section{DISCUSSION}

In the study, the majority of the samples from which S. paucimobilis was isolated were urine samples (31\%) followed by blood (24\%) and lower respiratory tract specimens $(16 \%)$. However, UTI was reported

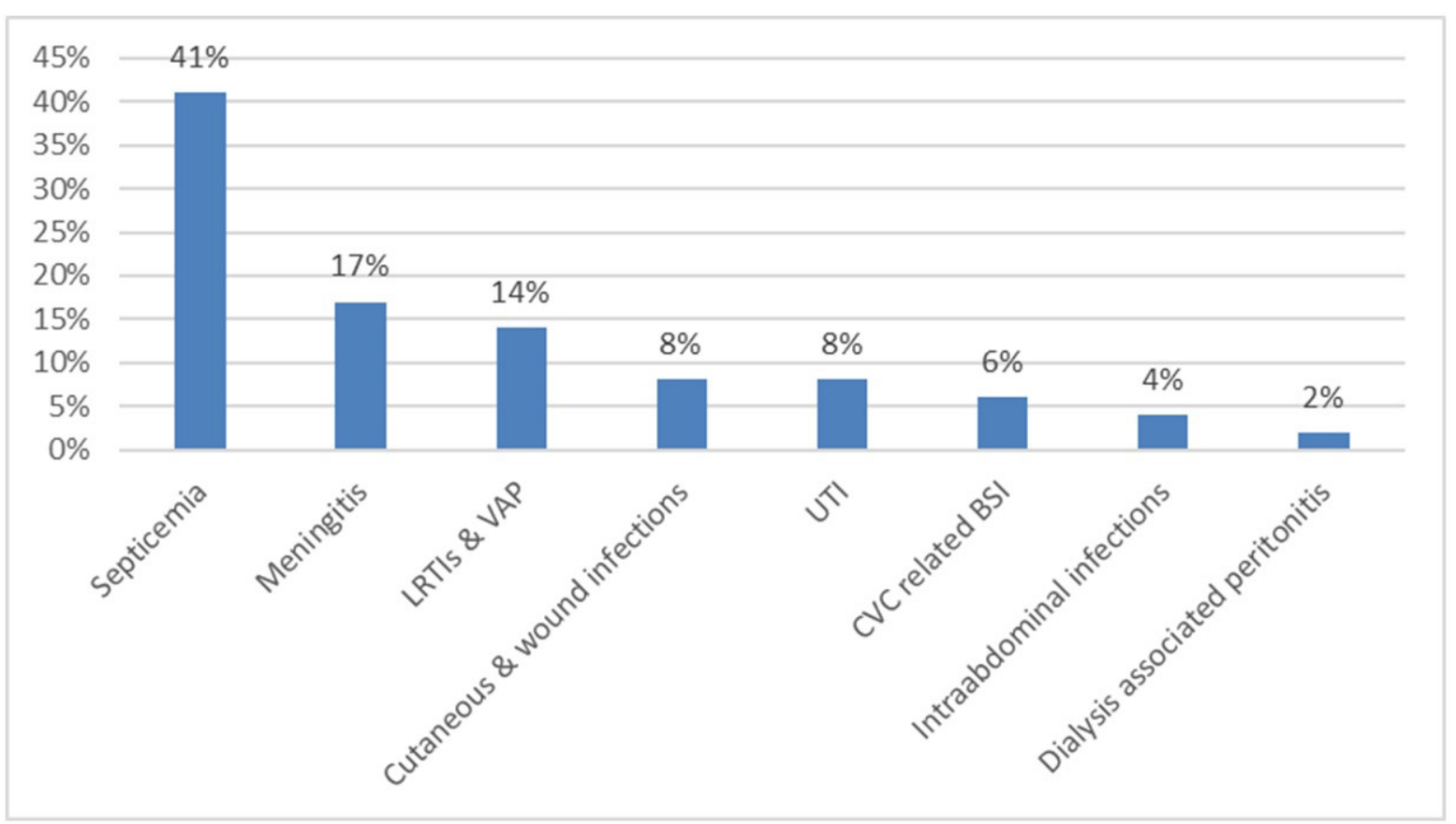

Fig. 1. Frequency distribution of clinical manifestations of the patients $(n=49)$

Table 2. Distribution of samples from which Sphingomonas paucimobilis was isolated $(\mathrm{n}=49)$

\begin{tabular}{lc}
\hline Samples & $\begin{array}{c}\text { Isolation of Sphingomonas } \\
\text { paucimobilis in \% (n=49) }\end{array}$ \\
\hline Urine & $31 \%(15 / 49)$ \\
Blood & $24 \%(12 / 49)$ \\
Endotracheal aspirate/ Endotracheal tip/suction tips/Bronchoalveolar lavage/ Sputum & $16 \%(8 / 49)$ \\
Pus/wound swabs & $12 \%(6 / 49)$ \\
Cerebrospinal fluid & $8 \%(4 / 49)$ \\
Sterile fluid (Peritoneal fluid/Pleural fluid/Ascitic fluid) & $8 \%(4 / 49)$ \\
\hline
\end{tabular}


RANJANA ROHILLA ET AL.

Table 3. Ward-wise distribution of isolates

\begin{tabular}{lc}
\hline Ward & Percentage of isolates (n=49) \\
\hline Intensive care units (ICUs) and High dependency Units (HDUs) & \\
ICUs HDUs & $37 \%(18 / 49)$ \\
Wards & $14 \%(7 / 49)$ \\
Medicine & \\
Surgery & $12 \%(6 / 49)$ \\
Nephrology & $8 \%(4 / 49)$ \\
Cardiothoracic Vascular surgery (CTVS) & $6 \%(3 / 49)$ \\
Obstetrics \& Gynecology & $4 \%(2 / 49)$ \\
Pediatrics & $2 \%(1 / 49)$ \\
Respiratory & $2 \%(1 / 49)$ \\
Outpatient departments & $2 \%(1 / 49)$ \\
Outpatient departments & $12 \%(6 / 49)$ \\
\hline
\end{tabular}

Table 4. Underlying predisposing conditions in patients $(n=49)$

\begin{tabular}{lcc}
\hline Underlying conditions & Percentage (Number) & P-value \\
\hline Diabetes & $22 \%(11 / 49)$ & $0.008725(\mathrm{~s})^{*}$ \\
Steroid use & $16 \%(8 / 49)$ & $0.00001(\mathrm{~s})$ \\
Malignancy & $8 \%(4 / 49)$ & $0.00001(\mathrm{~s})$ \\
Chronic renal failure or end-stage renal disease & $6 \%(3 / 49)$ & $0.083699(\mathrm{~ns}))^{* *}$ \\
Central venous catheter in-situ & $6 \%(3 / 49)$ & $<0.00001(\mathrm{~s})$ \\
Arthritis & $2 \%(1 / 49)$ & $0.127686(\mathrm{~ns})$ \\
Alcoholism & $2 \%(1 / 49)$ & $0.110655(\mathrm{~ns})$ \\
Chronic obstructive pulmonary disease & $2 \%(1 / 49)$ & $0.77744(\mathrm{~ns})$ \\
Acquired immunodeficiency syndrome (AIDS) & $2 \%(1 / 49)$ & $0.93382(\mathrm{~ns})$ \\
No predisposing factors & $33 \%(16 / 49)$ & $<0.00001(\mathrm{~s})$ \\
\hline
\end{tabular}

*s: significant $\quad * *$ ns: not significant

in $8 \%$ of patients from whom S. paucimobilis was isolated and all of the patients with UTI had associated co-morbidities. There are few documented reports suggesting UTI caused by $S$. paucimobilis and most of them had underlying conditions like malignancy, diabetes mellitus, and renal transplantation $(11,12)$. Moist hospital environment aids the survival of Sphingomonas spp. Contact with a contaminated medical device, catheters, implants, etc. can result in colonization and development of infection in the immunocompromised patients (11).

The majority of the patients in our study $(41 \%)$ were reported to have bloodstream infections caused by $S$. paucimobilis followed by meningitis (17\%) and lower respiratory tract infections (14\%). Similar reports of S. paucimobilis as an etiological agent of bacteremia have also been reported by lugito, Puca E et al. $(7,13)$. In fact, cases of community-acquired primary bacteremia have been reported by Puca E et al. (13). It has been isolated from blood in most cases presented in the literature. S. paucimobilis bacteremia has been reported mainly in patients with indwelling devices or immunocompromised hosts, especially those with neutropenia (14). In 2017, Goker T et al. have also reported $S$. paucimobilis from CSF samples (2). The patient presented with dizziness and headache and S. paucimobilis was subsequently isolated from CSF (15). The second case of meningitis in the literature was the one report by Tai and Velayuthan (16). It may also cause both nosocomial and community-acquired pneumonia with a mortality potential as was evident also in our study where $14 \%$ of patients also 
Table 5. Antibiotic susceptibility pattern of isolates to various antibiotics agents (as per MIC breakpoints for non-fermenters given in CLSI 2021) $(\mathrm{n}=49)$

\begin{tabular}{lcc}
\hline & Sensitive (\%) & Resistant (\%) \\
\hline Amikacin & $29(59 \%)$ & $20(41 \%)$ \\
Gentamicin & $29(59 \%)$ & $20(41 \%)$ \\
Aztreonam & $29(59 \%)$ & $20(41 \%)$ \\
Piperacilln-tazobactam & $29(59 \%)$ & $20(41 \%)$ \\
Ticarcillin clavulinic acid & $37(76 \%)$ & $12(24 \%)$ \\
Cefoperazone sulbactam & $37(76 \%)$ & $12(24 \%)$ \\
Ceftazidime & $35(72 \%)$ & $14(28 \%)$ \\
Ceftriaxone & $40(81 \%)$ & $9(19 \%)$ \\
Cefepime & $42(86 \%)$ & $7(14 \%)$ \\
Meropenem & $33(67 \%)$ & $16(33 \%)$ \\
Imipenem & $32(65 \%)$ & $17(35 \%)$ \\
Minocycline & $28(57 \%)$ & $21(43 \%)$ \\
Tigecycline & $35(72 \%)$ & $14(28 \%)$ \\
Ciprofloxacin & $29(59 \%)$ & $20(41 \%)$ \\
Levofloxacin & $36(74 \%)$ & $13(26 \%)$ \\
Colistin & $15(39 \%)$ & $30(61 \%)$ \\
\hline
\end{tabular}

Table 6. Antibiotic resistance patterns in Critical and Non-critical areas

\begin{tabular}{lcc}
\hline & MDR $(\mathbf{n}=\mathbf{1})$ & Non-MDR $(\mathbf{n}=\mathbf{4 8})$ \\
\hline Critical areas $(\mathrm{n}=25)$ & 1 & 24 \\
Non-critical areas $(\mathrm{n}=24)$ & 0 & 24 \\
& $\mathbf{X D R}(\mathbf{n}=\mathbf{1 3})$ & Non-XDR $(\mathbf{n}=\mathbf{3 6})$ \\
Critical areas $(\mathrm{n}=25)$ & 9 & 16 \\
Non-critical areas $(\mathrm{n}=24)$ & 4 & 20 \\
\hline
\end{tabular}

had features of pneumonia and empyema (16).

$43 / 49(87.75 \%)$ isolates were cultured from hospitalized patients and 6/49 (12\%) from outpatient departments. 25/49 (51\%) were isolated from patients admitted in ICUs and HDUs whereas the rest 18/49 (36.73\%) from patients admitted in different wards. Community-acquired infections by $S$. paucimobilis are not uncommon and have been reported in studies by Toh et al. and Puca $(13,14)$. In fact, their study reported a higher percentage of community-acquired S. paucimobilis infections compared with other studies (17). The environmental presence of $S$. paucimobilis in water and soil and the demonstration of any association between community-acquired $S$. paucimobilis infection and environmental exposure warrants additional studies (18). Increased chances of isolation of S. paucimobilis from hospital settings have also been reported in case studies by Goker T (2). In hospital settings, as was found in the current study, S. paucimobilis might have originated from devices such as indwelling catheters, ventilator and hemodialysis devices, nebulizer, sterile intravenous fluid, and contaminated hospital tap and distilled water (14).

Diabetes mellitus (22\%), steroid usage (16\%), and malignancy $(8 \%)$ were the most common co-morbid conditions observed in our study ( $\mathrm{P}$ value significant<0.05). In fact, a significant statistical association was found between the presence of underlying associated medical conditions and the isolation of $S$. paucimobilis. Similar findings have also been reported in a case study by Hardjo and a study by Toh (7, 14). Another case study by Puca also reports diabetes mellitus as the most commonly identified risk factor; advanced age, malignancy, immunosuppression, being the other attributing factors (14). The plausible reason for diabetes being a significant risk factor is that that infection results in inflammation along with activated innate immunity, which indirectly leads to insulin resistance. Also, malignancies lead to compromised immune system making patients more prone to transient infections by a nosocomial pathogen. $(19,20) S$. paucimobilis has been acknowledged to cause infection in the immunocompromised host with an increased propensity to infect patients with underlying conditions, hence can potentially survive and thrive in hospital environment (11).

Third and fourth generation cephalosporins like ceftriaxone $(81 \%)$ and cefepime $(86 \%)$ were found to be the most susceptible drugs whereas $61 \%$ of isolates were resistant to colistin followed by $43 \%$ of isolates demonstrating resistance to minocycline. Barring colistin, $>50 \%$ sensitivity of isolates was seen for the commonly used drugs for this infection however the increased resistance rates $(41 \%)$ to drugs like aminoglycosides, aztreonam, piperacillin -tazobactam, and fluoroquinolones like ciprofloxacin cannot be ignored and reiterate the fact that this organism also has the capability to become multidrug resistant in the near future if the currently available drugs are not used judiciously. As there are no definitive guidelines for antibiotic treatment for S. paucimobilis infections, individualized antibiotic therapy is initiated according to the in-vitro susceptibility pattern of the clinical isolate $(14,21)$.

Generally, S. paucimobilis is susceptible to carbapenems, aminoglycosides, trimethoprim-sulfame- 
thoxazole, and piperacillin/ tazobactam and resistant to penicillins and first-generation cephalosporins. The resistance to penicillins and first-generation cephalosporins is attributable to the production of chromosomally encoded beta-lactamase (7). A study by Toh has reported resistance to amikacin, ceftazidime, and fluoroquinolones, while in another study by ozdemir it was reported to be resistant to cefoxitin and ceftazidime $(14,21)$. In our study, $72 \%$ of isolates demonstrated a susceptible profile for ceftazidime. $90 \%$ of the sphingomonas isolates were Multidrug resistant (MDR) in a study from Ghana (22). Antimicrobial susceptibility variations have also been reported from many other research works wherein most strains were found to be resistant to beta-lactam drugs and the antimicrobial drugs like quinolones, carbapenems, beta-lactam/beta-lactamase inhibitors, and aminoglycosides were found to be effective $(5,11)$.

\section{CONCLUSION}

S. paucimobilis is an emerging infectious agent that is ubiquitous in natural environments but may also be isolated from the hospital milieu and holds importance in immunocompromised patients and those having other underlying comorbidities. Even in immune-competent patients it cannot be neglected. It can be an etiological agent for both nosocomial and community-acquired infections. Although prophylactic therapy can eliminate it, sensitivity patterns, however, should be categorically studied to establish an optimal therapy.

\section{REFERENCES}

1. Bavaro DF, Mariani MF, Stea ED, Gesualdo L, Angarano, Carbonara S. Sphingomonas paucimobilis outbreak in a dialysis room: case report and literature review of an emerging healthcare associated infection. Am J Infect Control 2020; 48: 1267-1269.

2. Goker T, Aşik RZ, Muhammet Bahadir Yilmaz, Çelik I, Tekiner A, Sphingomonas Paucimobilis: a rare infectious agent found in cerebrospinal fluid. $J$ Korean Neurosurg Soc 2017; 60: 481-483.

3. Bayram N, Devrim L, Apa H, Gulfidan G, Turkyilmaz HN, Guynay L. Spingomonas paucimobilis infection in children: 24 case reports. Mediterr J Hematol Infect
Dis 2013; 5(1): e2013040.

4. Ryan MP, Adley CC. Sphingomonas paucimobilis: a persistent gram negative nosocomial infectious organism. J Hosp Infect 2010; 75: 153-157.

5. Lin JN, Lai CH, Chen YH, Lin HL, Huang CK, Chen $\mathrm{WF}$, et al. Sphingomonas paucimobilis bacteremia in humans: 16 case reports and a literature review. $J$ Microbiol Immunol Infect 2010; 43: 35-42.

6. Walayat S, Malik A, Hussain N, Lynch T. Sphingomonas paucimobilis presenting as acute phlebitis: a case report. IDCases 2017; 11: 6-8.

7. Hardjo Lugito NP, Cucunawangsih, Kurniawan A. A lethal case of Sphingomonas paucimobilis bacteremia in an immunocompromised patient. Case Rep Infect Dis 2016; 2016: 3294639.

8. Gursoy S, Yasar KK, Sari ND, Kuvat N, Ozturk S (2018) Sphingomonas paucimobilis bacteremia in a hemodialysis patient and literature review. Int J Crit Care Emerg Med 2018; 4: 041.

9. Dsouza A, Mallepally AR, Marathe NA, Das K, Mohaptra B. A rare case of Sphingomonas paucimobilis spondylodiscitis managed surgically. J Orthop Case Rep 2021; 11: 91-96.

10. Clinical and Laboratory Standards Institute (CLSI). Performance standards for antimicrobial susceptibility testing. $30^{\text {th }}$ ed. CLSI supplement M100-S30. Wayne, PA: Clinical and Laboratory Standards Institute; 2020.

11. Demir T, Dadali M. Recurrent complicated urinary tract infection due to rare pathogen Sphingomonas paucimobilis: contamination or real deal? Infez Med 2016; 24: 241-244.

12. Lin JN, Lai CH, Chen YH, Lin HL, Huang CK, Chen WF, et al. Sphingomonas paucimobilis bacteremia in humans: 16 case reports and a literature review. $J$ Microbiol Immunol Infect 2010; 43: 35-42.

13. Puca E, Harxhi A, Mehmeti J, Rroji A, Huti G, Jata B, et al. Sphingomonas paucimobilis from blood stream infection to spondylodiscitis. Clin Microbiol 2015; 4(3): e1000203.

14. Toh HS, Tay HT, Kuar WK, Weng TC, Tang HJ, Tan CK. Risk factors associated with Sphingomonas paucimobilis infection. J Microbiol Immunol Infect 2011; 44: 289-295.

15. Mehmood H, Khan N, Ullah S, Ullah A, Marwat A. A rare case of Sphingomonas paucimobilis meningitis in the absence of cerebrospinal fluid pleocytosis. J Investig Med High Impact Case Rep 2018; 6: 2324709618756424.

16. Tai ML, Velayuthan RD. Sphingomonas paucimobilis: an unusual cause of meningitis-case report. Neurol Med Chir (Tokyo) 2014; 54: 337-340.

17. Cheong HS, Wi YM, Moon SY, Kang CI, Son JS, Ko $\mathrm{KS}$, et al. Clinical features and treatment outcomes 
of infections caused by Sphingomonas paucimobilis. Infect Control Hosp Epidemiol 2008; 29: 990-992.

18. Dervisoglu E, Meric M, Kalender B, Sengul E. Sphingomonas paucimobilis peritonitis: a case report and literature review. Perit Dial Int 2008; 28: 547-550.

19. Jeon CY, Furuya EY, Smaldone A, Larson EL. Post-admission glucose levels are associated with healthcare-associated bloodstream infections and pneumonia in hospitalized patients with diabetes. $J$ Diabetes Complications 2012; 26: 517-521.

20. Titmarsh GJ, McMullin MF, McShane CM, Clarke M,
Engels EA, Anderson LA. Community-acquired infections and their association with myeloid malignancies. Cancer Epidemiol 2014; 38: 56-61.

21. Özdemir M, Pekcan S, Demircili ME, Taşbent FE, Feyzioğlu B, Pirinç Ş, et al. A rare cause of bacteremia in a pediatric patient with Down syndrome: Sphingomonas paucimobilis. Int J Med Sci 2011; 8: 537-539.

22. Agyepong N, Govinden U, Owusu-Ofori A, Essack SY. Multidrug-resistant gram-negative bacterial infections in a teaching hospital in Ghana. Antimicrob Resist Infect Control 2018; 7: 37. 